\title{
Takotsubo cardiomyopathy caused by emotional stress associated with the Coronavirus Disease 2019 (COVID-19) pandemic
}

\author{
Devika Kir ${ }^{1}$, Nusen Beer ${ }^{1}$, and Eduardo de Marchena ${ }^{1}$ \\ ${ }^{1}$ University of Miami Miller School of Medicine
}

August 22, 2020

\begin{abstract}
The COVID-19 pandemic has had far-reaching consequences beyond the disease itself, including economic, social, political, religious and psychological implications. This novel coronavirus has been shown to have cardiovascular manifestations in the form of arrhythmias, conduction disturbances, myocarditis, stress cardiomyopathy, myocardial injury and myocardial ischemia or infarction from increased microvascular and/or macrovascular coagulopathy. However, in addition to these direct effects, we are now starting to recognize indirect cardiovascular effects of COVID-19 in the form of increased incidence of Takutsobo cardiomyopathy in patients without any evidence of coronavirus infection. In this case series, we present two post-menopausal women, presenting with chest pain and acute coronary syndrome, who are finally diagnosed with stress cardiomyopathy, triggered by increased emotional stress related to the pandemic. There is data from a retrospective cohort analysis showing a four-fold increase in the incidence of acute coronary syndrome resulting from stress cardiomyopathy during the pandemic period compared to similar times periods before the pandemic. Hence, health care providers need to cognizant of the emotional ramifications of the ongoing pandemic in the form of increased risk of Takotsubo cardiomyopathy. Moreover, urgent measures need to be taken to help at-risk population cope with the ongoing stressors to help decrease the incidence of this cardiomyopathy.
\end{abstract}

\section{Introduction:}

The World Health Organization declared COVID-19 a pandemic on March 11, 2020 (1). As of mid-August 2020, there have been about 21,250,743 confirmed cases worldwide with 766,648 deaths reported across the globe, based on the Center for Systems Science and Engineering at Johns Hopkins University (2). This novel coronavirus has been shown to have cardiovascular manifestations in the form of arrhythmias (3), conduction disturbances (4), myocarditis (5), stress cardiomyopathy (6), myocardial injury (7) and myocardial ischemia or infarction from increased microvascular and/or macrovascular coagulopathy $(8,9)$. However, in addition to these direct effects, we are now starting to recognize indirect cardiovascular effects of COVID-19 in the form of increased incidence of Takutsobo cardiomyopathy in patients without any evidence of coronavirus infection. Based on a retrospective cohort analysis from Ohio, acute coronary syndrome resulting from stress cardiomyopathy was found to be much higher during the pandemic (about 8\%) period, compared to similar times periods before the pandemic (about 1.5-2\%) (10). This increase in stress cardiomyopathy is likely resulting from increased emotional and psychological stress related to social isolation, which is further exacerbated by the economic consequences of the shut-down. In this article, we present a case series of two such patients diagnosed with stress cardiomyopathy caused by psychological trauma due to the ongoing pandemic.

\section{Methods:}

This case series is exempt by our Institutional Review Board (IRB). Informed oral consent was obtained from both the patients mentioned in this case series.

\section{Case 1:}


An 85-year old Hispanic woman with past medical history significant for hypertension, pre-diabetes, chronic kidney disease, hyperlipidemia, presented to the hospital with acute onset chest pain, that started 2 hours prior to presentation. The pain was described as a diffuse pressure like sensation, radiating to her jaw and back, was waxing and waning in severity, but never fully resolved. She denied any associated nausea, vomiting, leg swelling, dizziness, syncope or shortness of breath. She did report increased palpitations in the last 2-3 weeks, but were not associated with her chest pain. She denied any antecedent fevers, chills, myalgias, sick contacts or recent travel.

Chest pain had intensified about an hour prior to arrival, when she called emergency medical services. ST-segment Elevation Myocardial Infarction (STEMI) alert was called in field for possible lateral wall ST segment changes. On arrival to the emergency department, our patient was afebrile with temperature of 36.9 degrees Celsius, tachycardic to 120 beats per minute, her blood pressure was $108 / 45 \mathrm{~mm} \mathrm{Hg}$ (with no significant difference between right and left arm), she was mildly tachypneic at 15 breaths per minute, and oxygen saturation was 95 percent on room air. Physical exam was remarkable for an elderly, anxious, ill-appearing, frail woman, who was tachycardic, with normal pulses, normal cardiac exam with no significant murmur or rub on auscultation, lungs were clear to auscultation.

Electrocardiogram (EKG) on arrival (Figure 1), showed sinus tachycardia with inferior Q waves, poor R wave progression and non-specific ST-segment changes. She was loaded with aspirin $325 \mathrm{mg}$ and clopidogrel 600 mg given concern for acute coronary syndrome, STEMI alert was canceled with plan for invasive assessment pending nasopharyngeal antigen testing for Severe Acute Respiratory Syndrome Coronavirus 2 (SARS-CoV2). Initial labs showed a normal leukocyte count of 7700 cells/uL with a normal differential, normocytic anemia with hemoglobin of $10 \mathrm{~g} / \mathrm{dL}$ with a normal platelet count of 207,000 cells/uL, chemistry was notable for elevated creatinine to $1.57 \mathrm{mg} / \mathrm{dL}$ (with estimated glomerular filtration rate of $30 \mathrm{~mL} / \mathrm{min} / 1.73 \mathrm{~m}^{2}$ ) and mildly elevated urea nitrogen at $28 \mathrm{mg} / \mathrm{dL}$, electrolytes; while coagulation parameters and liver function tests were within normal limits. Cardiac biomarkers were elevated; initial troponin $\mathrm{T}$ was elevated at 1.31 $\mathrm{mg} / \mathrm{mL}$ (reference range 0-0.06) and NT-proBNP was elevated at 3,397 $\mathrm{pg} / \mathrm{mL}$ (reference range 0-450). Test was SARS-CoV-2 was negative and patient was taken for urgent coronary angiography. Coronary angiogram was negative for any significant obstructive coronary artery disease, left ventriculogram showed an ejection fraction of $45-50 \%$, with severe hypokinesis of the apical segments with apical ballooning and basal hyperkinesis, changes consistent with classical Takotsubo cardiomyopathy (Figure 2).

On further questioning, our patient endorsed being in extreme emotional distress because of COVID social isolation, and her anxiety was further exacerbated by the recent political demonstrations/riots for racial justice. She was monitored in our coronary care unit for 48 hours, was started on beta-blockers, anxiolytics and afterload reduction with good effect. No significant arrhythmias were noted and she continued to be hemodynamically stable. Psychology was consulted for psychosocial assessment and coping skills were taught and information on community resources was provided. She was subsequently discharged home with close cardiology follow-up.

\section{Case 2:}

A 70-year old healthy Hispanic woman with past medical history significant only for hypertension, presented with new-onset severe retrosternal chest discomfort, that was worse with activity, associated with diaphoresis and shortness of breath, symptoms started about 24 hours prior to presentation. She was seen at her cardiologist's office, her EKG showed new deep T wave inversions in the precordial leads and subtle ST elevation in the inferior leads (Figure 3). She was sent to the emergency department from the office, her echocardiogram showed severe hypokinesis of the antero-apical wall, confirming anterior myocardial infarction. She tested negative for SARS-CoV-2 and was emergently taken to the cardiac catheterization lab for further evaluation.

Coronary angiography revealed only mild calcification and non-obstructive disease in the coronary arteries with a right dominant circulation (Figure 4). Myocardial bridge was noted in the proximal-mid left anterior descending artery (Figure 4). Further, left ventricular end-diastolic pressure was elevated at $22 \mathrm{~mm} \mathrm{Hg}$ and left ventriculography showed a severely depressed left ventricular ejection fraction of $30 \%$ with antero- 
apical and infero-apical wall akinesis (Figure 4 and Supplementary videos 1 and 2). Overall, her clinical presentation was hence consistent with Takotsubo cardiomyopathy. She was started on medical therapy with beta-blockers, anxiolytics, and discharged home with close cardiology follow-up.

In retrospect, during our interaction with the patient, she endorsed significant stress during the past few weeks, due to ongoing health issues. She had noticed a gradual decline in her visual acuity, however, due to the ongoing pandemic and outpatient appointment restrictions due to that, she was not able to schedule an ophthalmology appointment, despite repeated attempts, further adding to her stress.

\section{Conclusions:}

Stress cardiomyopathy or Takotsubo cardiomyopathy is characterized by transient left ventricular dysfunction in response to an emotional or physical stressor (11). Stress cardiomyopathy has been implicated in about 1-2\% of patients diagnosed with acute coronary syndromes. Among different demographic groups, postmenopausal women are most frequently affected, which is attributed to increased prevalence of co-morbid depression, anxiety, sleep disturbances and an increased sympathetic drive in this population (11).

The ongoing viral pandemic has had far-reaching consequences on the physical, economical and emotional well-being of millions of individuals across the world. A recent retrospective study confirmed an increase in the incidence of stress cardiomyopathy during the pandemic, about four-fold, compared to pre-pandemic era, likely due to increased emotional stress from the spreading pandemic, deaths and economic crisis (10). This cardiomyopathy has been shown to occur in patients who tested negative for the novel coronavirus and hence, is independent of COVID's effect on the cardiovascular system.

In this case series, we present two such cases of Takotsubo cardiomyopathy triggered by heightened emotional stress during the pandemic, in the most vulnerable demographic group- post-menopausal women. While the first patient lived along, with family in a distant state and suffered from worsening of her anxiety due to quarantine and social distancing, the second patient presented in this case series had increased emotional stress because of health issues and finding it difficult to navigate the health system during the pandemic. Both fell prey to stress cardiomyopathy, as an indirect consequence of the ongoing pandemic. In addition to the stress related to the pandemic, ongoing political tensions and demonstrations related to racial inequality, may have contributed.

Stress cardiomyopathy can be associated with in-hospital mortality as high as $5 \%$, with complications ranging from cardiogenic shock, left ventricular outflow obstruction, malignant arrythmias, systemic thromboembolism and rarely ventricular rupture (11). With the vaccine for SARS-CoV-2 virus still not in immediate sight, the time-course for this pandemic is unpredictable. With the essential social distancing measures in place, elderly population may not have adequate family support in these difficult times and are at an increased risk for stress- related comorbidities. Further, in addition to direct cardiovascular effects of the novel coronavirus in the form of conduction disturbances, arrhythmias, myocarditis, atherosclerotic plaque rupture, coronary and pulmonary emboli; health care providers need to cognizant of the emotional ramifications of the ongoing pandemic in the form of increased risk of Takotsubo cardiomyopathy. Hence, urgent measures need to be taken to help at-risk population cope with the ongoing stressors, provide psychiatric care and psychological counseling through tele-health, to help decrease the incidence of this cardiomyopathy.

\section{Author Contributions:}

This study was designed and conceptualized by D. Kir and EJ De Marchena. D. Kir, N. Beer and EJ De Marchena were involved in writing and critical review of the manuscript for accuracy and intellectual content.

\section{References:}

1. TIME. World Health Organization Declares COVID-19 a 'Pandemic'. 2020 [Available from: https://time.com/5791661/who-coronavirus-pandemic-declaration/.

2. JHU. COVID-19 Dashboard 2020 [Available from: https://coronavirus.jhu.edu/map.html. 
3. Bhatla A, Mayer MM, Adusumalli S, Hyman MC, Oh E, Tierney A, et al. COVID-19 and cardiac arrhythmias. Heart Rhythm. 2020.

4. Kir D, Mohan C, Sancassani R. HEART BRAKE-An unusual cardiac manifestation of Coronavirus disease 2019 (COVID-19). JACC Case Rep. 2020.

5. Besler MS, Arslan H. Acute myocarditis associated with COVID-19 infection. Am J Emerg Med. 2020.

6. Minhas AS, Scheel P, Garibaldi B, Liu G, Horton M, Jennings M, et al. Takotsubo Syndrome in the Setting of COVID-19 Infection. JACC Case Rep. 2020.

7. Bonow RO, Fonarow GC, O'Gara PT, Yancy CW. Association of Coronavirus Disease 2019 (COVID-19) With Myocardial Injury and Mortality. JAMA Cardiol. 2020.

8. Guagliumi G, Sonzogni A, Pescetelli I, Pellegrini D, Finn AV. Microthrombi and ST-Segment Elevation Myocardial Infarction in COVID-19. Circulation. 2020.

9. Bangalore S, Sharma A, Slotwiner A, Yatskar L, Harari R, Shah B, et al. ST-Segment Elevation in Patients with Covid-19 - A Case Series. N Engl J Med. 2020;382(25):2478-80.

10. Jabri A, Kalra A, Kumar A, Alameh A, Adroja S, Bashir H, et al. Incidence of Stress Cardiomyopathy During the Coronavirus Disease 2019 Pandemic. JAMA Netw Open. 2020;3(7):e2014780.

11. Medina de Chazal H, Del Buono MG, Keyser-Marcus L, Ma L, Moeller FG, Berrocal D, et al. Stress Cardiomyopathy Diagnosis and Treatment: JACC State-of-the-Art Review. J Am Coll Cardiol. 2018;72(16):1955-71.

Figure Legends:

Figure 1 Electrocardiogram on arrival shows sinus tachycardia, with inferior Q waves, poor R wave progression and non-specific ST-segment changes

Figure 2a) Coronary angiogram showing a right dominant circulation without any obstructive disease noted in the right coronary artery $2 \mathrm{~b}$ ) Antero-posterior cranial view showing bifurcation of left main into left anterior descending and circumflex arteries without any obstructive coronary artery disease noted throughout the length of left anterior descending artery 2c) Antero-posterior caudal view showing absence of obstructive coronary artery disease in the circumflex territory 2d) Left ventriculogram showing severe hypokinesis of the apical segments with apical ballooning and basal hyperkinesis, changes consistent with classical Takotsubo cardiomyopathy.

Figure 3 Electrocardiogram on arrival shows sinus rhythm symmetric deep T wave inversions in the precordial leads and inferior ST changes

Figure 4a) Coronary angiogram showing a right dominant circulation without any significant disease in the right coronary artery 4b) Antero-posterior cranial view showing a myocardial bridge in the mid-left anterior descending artery without any obstructive coronary artery disease 4c) Left anterior oblique cranial view showing bifurcation of the left main in left anterior descending and circumflex arteries, with no significant obstructive disease noted in the circumflex distribution 4d) Left ventriculogram showing severe hypokinesis of the apical segments with apical ballooning and basal hyperkinesis during systole, changes consistent with classical Takotsubo cardiomyopathy

Supplementary Video 1 Parasternal long-axis view of the echocardiogram for the second case shows preserved basal wall motion and apical hypokinesis.

Supplementary Video 2 Apical three-chamber view of the echocardiogram for the second case confirms apical and mid-apical antero-septal hypokinesis. 

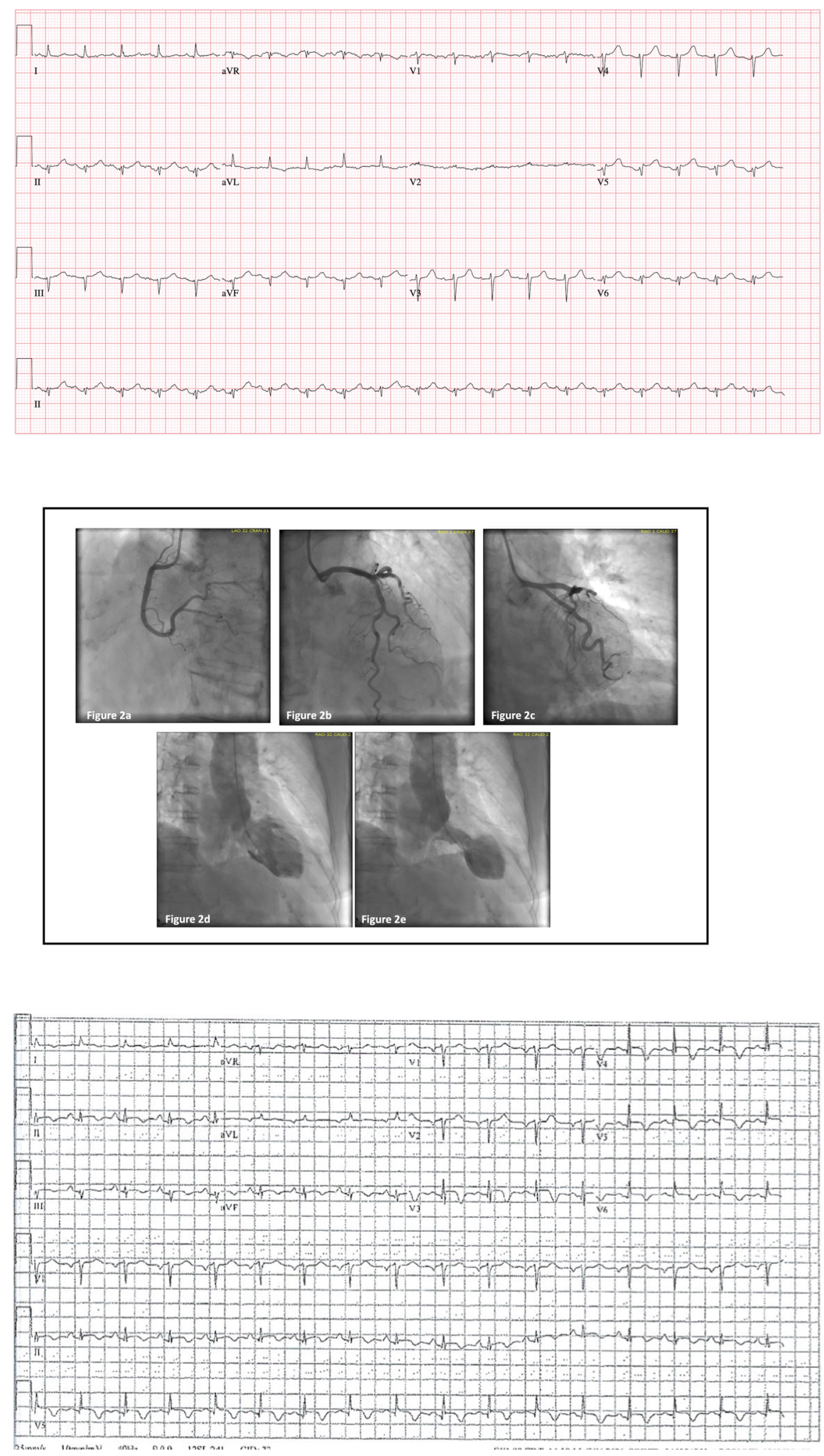


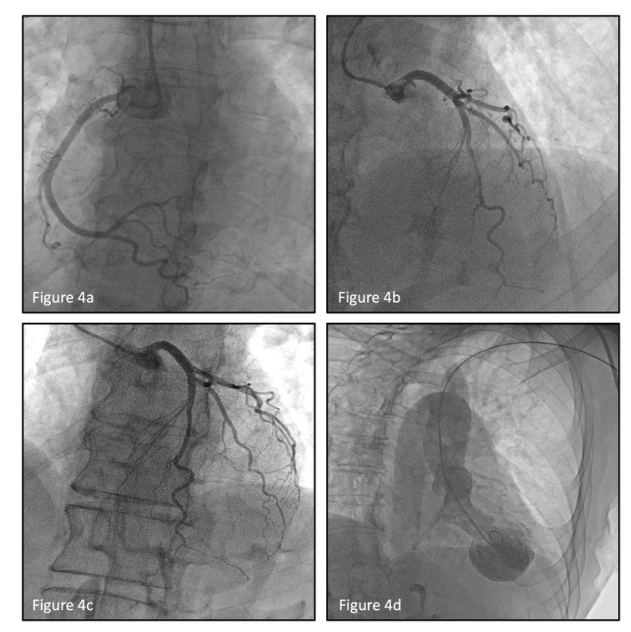

